\title{
Initial Experience With Radium-223 Chloride Treatment at the Kanazawa University Hospital
}

\author{
KAZUFUMI NAKASHIMA ${ }^{1}$, TOMOYUKI MAKINO ${ }^{1}$, SUGURU KADOMOTO $^{1}$, \\ HIROAKI IWAMOTO ${ }^{1}$, HIROSHI YAEGASHI ${ }^{1}$, MASASHI IIJIMA ${ }^{1}$, \\ SHOHEI KAWAGUCHI ${ }^{1}$, TAKAHIRO NOHARA ${ }^{1}$, KAZUYOSHI SHIGEHARA ${ }^{1}$, \\ KOUJI IZUMI ${ }^{1}$, YOSHIFUMI KADONO ${ }^{1}$, SHINRO MATSUO ${ }^{2}$ and ATSUSHI MIZOKAMI ${ }^{1}$ \\ ${ }^{1}$ Department of Integrative Cancer Therapy and Urology, Kanazawa \\ University Graduate School of Medical Science, Kanazawa, Japan; \\ ${ }^{2}$ Department of Nuclear Medicine Biotracer Medicine, \\ Kanazawa University Graduate School of Medical Science, Ishikawa, Japan
}

\begin{abstract}
Background/Aim: To evaluate our initial experience with radium-223 chloride (Ra-223). Materials and Methods: A total of 26 castration-resistant prostate cancer (CRPC) patients with bone metastases, treated with Ra-223 at our hospital were evaluated. This study aimed to observe adverse events (AEs) and changes in serum markers, and Bone Scan Index (BSI). Additionally, the relationship between these values and OS was investigated. Results: The observed AEs mainly included fatigue and nausea. Alkaline phosphatase $(A L P)$ and bone-type alkaline phosphatase (BAP) levels decreased following the treatment; however, those of PSA and 1-CTP tended to increase, regardless of Ra-223 administration. Overall survival (OS) was significantly improved in cases with a baseline BSI value of $<2$ compared with those with a baseline BSI value of $\geq 2$. Moreover, the decrease in BSI after administration of Ra-223 was an independent factor, significantly prolonging $O S$. Conclusion: ALP and BAP levels and BSI values are suitable evaluation markers during treatment with Ra-223. Also, baseline BSI values and the decrease in BSI following treatment are independent factors predicting $O S$.
\end{abstract}

Patients with castration-resistant prostate cancer (CRPC) have a low frequency of both hepatic and pulmonary metastases (approximately 20\%), but exhibit a high

Correspondence to: Atsushi Mizokami, MD, Ph.D., Department of Integrative Cancer Therapy and Urology, Kanazawa University Graduate School of Medical Science, 13-1 Takaramachi, Kanazawa, Ishikawa 920-8640, Japan. Tel: +81 762652393, Fax: +81 762344263,e-mail: mizokami@staff.kanazawa-u.ac.jp

Key Words: Alkaline phosphatase, Bone Scan Index, castrationresistant prostate cancer, prostate cancer, radium-223 chloride. frequency of bone metastasis $(84-93 \%)(1)$. Hence, it is crucial to treat bone metastasis. Furthermore, symptomatic skeletal events (SSEs) due to bone metastases are common complications in metastatic CRPC (mCRPC) (2). Bone metastasis and skeletal-related events are linked to shortened survival in mCRPC patients (3). These reports suggest that prevention of SSEs is important for the quality of life.

Radium-223 (Ra-223), is a calcium mimetic absorbed at the sites of ongoing bone metabolism (such as the location of bone metastases) and possesses antitumor properties. Firstly, Nilsson et al. demonstrated the therapeutic potential of Ra-223 in bone metastatic CRPC (4). Concerning the safety and efficacy of Ra223, the phase III Alpharadin in Symptomatic Prostate Cancer Patients (ALSYMPCA) trial involving CRPC patients with bone metastases demonstrated a significantly prolonged overall survival (OS) and time to the onset of SSEs in the Ra-223 group versus the placebo group (5). This study showed that Ra223 has a good tolerability profile, a low incidence of bone marrow suppression, and few adverse events (AEs) compared to the placebo group. Moreover, in contrast to the ALSYMPCA trial, Ra-223 can be administered to asymptomatic patients in an international early-access program (iEAP). An iEAP is a phase IIIb trial in which patients received a maximum of six intravenous injections of Ra-223 (55 kBq/kg every 4 weeks), resulting in a median OS of 16 months (95\% confidence interval [CI]: 13-not available), which showed a good therapeutic outcome (6). In a Phase II study involving Japanese CRPC patients with bone metastasis, Ra-223 was effective and well tolerated. The results showed that its efficacy and safety were comparable with those observed in the ALSYMPCA trial (7). Based on this Japanese trial, Ra-223 was approved in March 2016 and commercialized in June 2016 in Japan. It is essential to evaluate progression or improvement of bone metastasis following various treatments. In addition, determination of the extent of disease (EOD) using bone 
Table I. Patients' characteristics.

\begin{tabular}{lc}
\hline Number of patients & 26 \\
\hline Age & \\
Median (years) & 70 \\
$\quad>75$ & $8(30.8)$ \\
ALP (U/l) & $10(40)$ \\
$\quad<220$ & $15(60)$ \\
$220 \leq$ & \\
Bisphosphonate administration & $24(92.3)$ \\
$\quad$ Yes & $21(80.8)$ \\
Zoledronic acid & $3(11.5)$ \\
Denosumab & $2(7.7)$ \\
No & \\
History of docetaxel use & $12(46.2)$ \\
Yes & $14(53.8)$ \\
No & $16(69.6)$ \\
Concomitant medications & $7(30.4)$ \\
Yes & \\
No & $4(15.4)$ \\
History of Metastron & \\
Yes & $22(84.6)$ \\
No use & \\
EOD (Extent of disease) & 1 \\
$1 .(>6$ bone metastatic foci) & 5 \\
$2 .(6-20$ bone metastatic foci) & 17 \\
$3 .(20$ bone metastatic foci) & 2 \\
$4 .($ Superscan) & \\
\hline
\end{tabular}

Concomitant medications

Ethinyl estradiol: 13 patients

Enzalutamide: 3 patients

Abiraterone acetate: 2 patients

Steroid: 6 patients

Others: 2 patients

scintigraphy was proposed by Soloway et al. (8). Although EOD is still used for the evaluation of bone metastases, timedependent changes in the grade of EOD are too minute for monitoring the follow-up of bone metastases and are lacking in quantifiability. In contrast, a computer-assisted diagnosis software (EXINI bone ${ }^{\circledR}$ or BONENAVI ${ }^{\circledR}$ ), which calculates the degree of bone metastasis quantitatively (Bone Scan Index, BSI) and the number of bone metastasis automatically, was developed $(9,10)$. The creation of a database for Japanese patients using BONENAVI ${ }^{\circledR}$ resulted in increased specificity and improvement of diagnostic accuracy (11). BONENAVI ${ }^{\circledR}$ can trace a chronological change in the whole body. Therefore, the BSI can be a very useful index to monitor and follow-up on bone metastases (12). Furthermore, in CRPC patients with bone metastases, changes in the BSI following treatment reportedly correlate with prognosis $(13,14)$.

However, imaging evaluation of bone metastasis through Ra-223 treatment is currently not fully performed. The aim of this study was to observe the safety, changes in serum markers
Table II. Adverse events.

\begin{tabular}{lcccc}
\hline & $\begin{array}{c}\text { All } \\
\text { grades }\end{array}$ & $\%$ & $\begin{array}{c}\text { Grade } 3 \\
\text { or above }\end{array}$ & $\%$ \\
\hline $\begin{array}{l}\text { Nausea/Appetite reduction } \\
\text { (digestive problems) }\end{array}$ & 7 & 26.9 & 1 & 4.8 \\
$\begin{array}{l}\text { Dysgeusia } \\
\text { Diarrhea }\end{array}$ & 2 & 8.7 & 0 & 0 \\
Bone pain & 5 & 19.2 & 0 & 0 \\
Fatigue/Malaise & 3 & 11.5 & 1 & 4.8 \\
Laryngeal edema & 7 & 26.9 & 0 & 0 \\
Anemia & 1 & 3.8 & 0 & 0 \\
Thrombocytopenia & 2 & 7.6 & 0 & 0 \\
Neutropenia & 2 & 7.6 & 0 & 0 \\
Impaired liver function & 1 & 3.8 & 0 & 0 \\
\hline
\end{tabular}

Completed 6 cycles: 13 patients (50.0\%); $4-5$ cycles: 6 patients (23.1\%); $1-3$ cycles: 6 patients $(23.1 \%)$.

(prostate specific antigen [PSA], alkaline phosphatase [ALP], bone-type alkaline phosphatase [BAP], pyridinoline crosslinked carboxyterminal telopeptide of type 1 collagen [1CTP]) and BSI values, and the relationship between these values and OS in CRPC patients treated with Ra-223.

\section{Materials and Methods}

Patients. For this institutional review board-approved retrospective study at Kanazawa University Hospital, the medical records of 26 CRPC patients receiving Ra-223 treatment, who were followed between June 2016 and April 2018, were included. In this study, 26 CRPC patients with bone metastases (but no visceral metastases) received a maximum of six intravenous injections of Ra-223 (50 kBq/kg every 4 weeks) with standard androgen-deprivation therapy. Moreover, these patients were treated with androgen receptor axis target agents, ethinylestradiol, zoledronic acid, or denosumab.

Study design. The serum markers PSA, ALP, BAP, and 1-CTP were evaluated during Ra-223 treatment. In order to quantitatively evaluate the degree of bone metastasis, bone scintigraphy was conducted and the BSI was measured using the BONENAVI ${ }^{\circledR}$ software. AEs and others were also evaluated. AEs were reported using the National Cancer Institute Common Terminology Criteria for Adverse Events, version 4.03.

The primary aim of this study was to observe the percentage changes in ALP, PSA, BAP, and 1-CTP levels at 12 weeks following treatment. The levels of ALP, PSA, BAP and 1-CTP, along with the BSI values, were measured in all patients at the following measurement points: prior to treatment (considered as baseline), 4, 8, and 12 weeks after treatment, and after the final treatment. Measurement of ALP, PSA, BAP, and 1-CTP 4 weeks after the final treatment was defined as "After the final treatment". Regarding BSI, "After the final treatment" was defined as 4-12 weeks after the final treatment.

The secondary aim was to investigate the relationship between these values (ALP, PSA, BAP, 1-CTP and BSI) and OS in CRPC patients treated with Ra-223. We analyzed the relationship between these values and OS by univariate analysis and multivariate analysis. 
Statistical analyses. In this study, statistical analyses were performed using the SPSS statistical software package (version 22.0; SPSS Inc., Chicago, IL, USA). Changes in serum data and BSI values were evaluated using the Wilcoxon signed-rank test. The OS curve was estimated using the Kaplan-Meier method and differences were compared with the log-rank test. In all analyses, statistical significance was defined as a $p$-value of $<0.05$.

\section{Results}

Twenty-six patients treated with Ra-223 at the Kanazawa University Hospital between June 2016 and April 2018 were investigated. The median age of the patients was 70 years old. Fifteen patients $(60 \%)$ showed high levels of ALP prior to treatment (Table I). Half of the patients $(13 / 26,50 \%)$ completed six cycles of treatment (Table II). The vast majority (24 patients, 92.3\%) were treated with bisphosphonates and 13 patients $(50 \%)$ received concomitant treatment with ethinylestradiol. Three patients (11.5\%) received concomitant treatment with enzalutamide, two patients $(7.7 \%)$ received concomitant treatment with abiraterone acetate + prednisolone, and six patients $(23.1 \%)$ received concomitant treatment with steroid, etc. (Table I). AEs were reported in 16 patients $(61.5 \%)$ and major AEs, such as fatigue $(26.9 \%)$, nausea/appetite reduction $(26.9 \%)$, diarrhea (19.2\%), and bone pain (11.5\%), were observed regardless of the grades of AE (Table II). Grade $\geq 3$ AEs (bone pain; $n=1$, nausea/appetite reduction; $n=1$ ) were reported (Table II). Bone fractures at any site were not observed during the observation periods.

Twelve weeks after treatment, the percentage change in PSA levels increased by $60.3 \%$, regardless of Ra-223 administration (Figure 1A). Among patients with a decreased PSA, those treated with combination therapy occupied $80 \%$ (8/10 patients) (Figure 1A). PSA (PSA levels prior to treatment) significantly increased 4 weeks after the final treatment $(p<0.05$; Figure 1B). In contrast, 12 weeks after treatment, the percentage change in ALP levels decreased by $35.8 \%$ (Figure 2A). Among patients with decreased ALP, those treated with combination therapy occupied $70.8 \%(17 / 24$ patients) (Figure 2A). Ra-223 significantly decreased ALP and $\mathrm{BAP}$ at 4,8 , and 12 weeks, and 4 weeks after the final treatment (all $p<0.05$; Figure $2 \mathrm{~B}$ and Figure $3 \mathrm{~A}$, respectively)

Although the levels of the osteolytic marker 1-CTP did not change significantly until 12 weeks of treatment, they were elevated 4 weeks after the final treatment $(p<0.05$; Figure 3B).

In bone scintigraphy, the flare phenomenon (BSI flare) was observed between 8 and 12 weeks after treatment $(5 / 15$ patients; $33.3 \%)$ (Figure $4 \mathrm{~A})$. The BSI values declined after treatment (11/15 patients; 73.3\%). Among patients with decreased BSI, those treated with combination therapy occupied $63.6 \%$ (7/11 patients) (Figure 4B). The BSI did not significantly decline at the
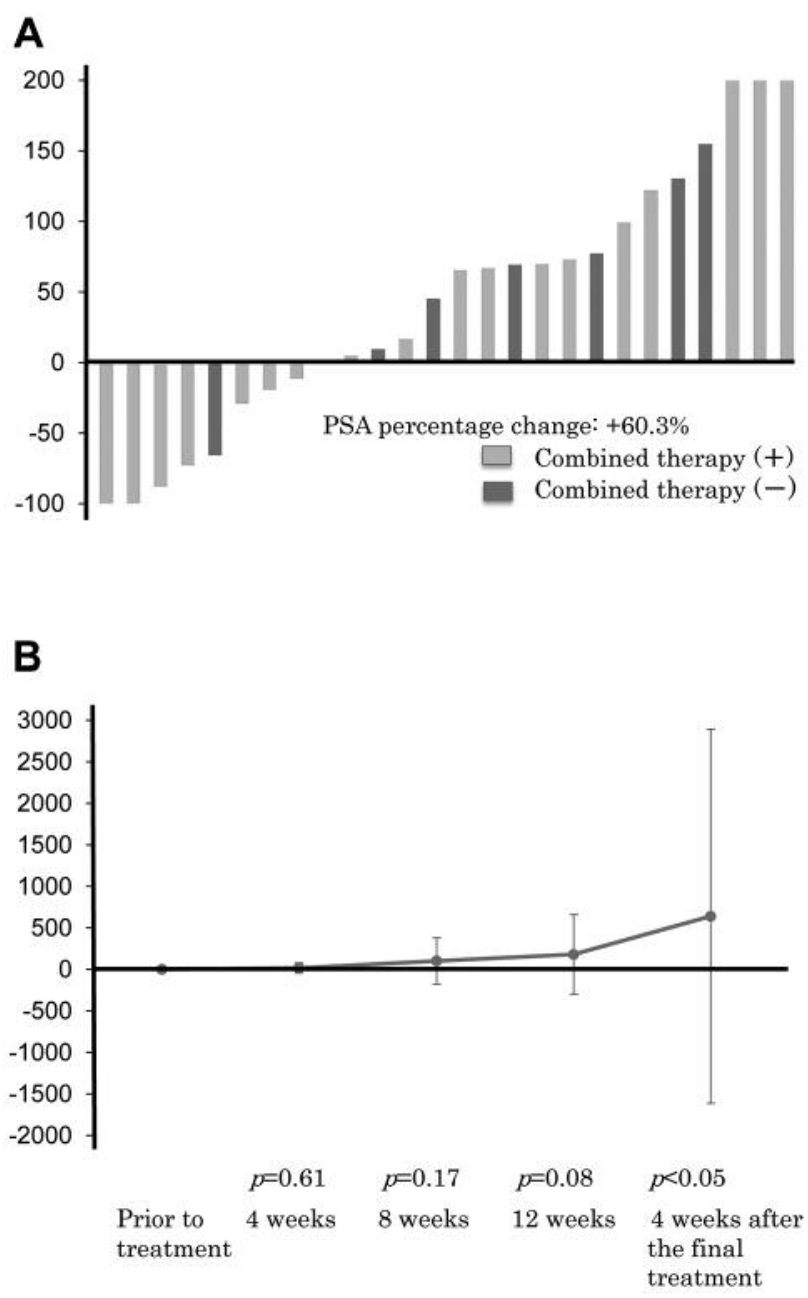

Figure 1. PSA changes from baseline with Ra-223 therapy. A: Percentage changes in PSA levels 12 weeks after treatment. Combined therapy was defined as concomitant treatment with "ethinylestradiol", "enzalutamide" and "abiraterone acetate + prednisolone". PSA: prostate specific antigen. $B$ : Percentage change from baseline PSA (mean levels of PSA $\pm S D$ ). PSA: Prostate specific antigen; SD: standard deviation.

midpoint of treatment. However, it declined significantly after treatment (Figure 4C). This result indicates that even if the flare occurred only once in this study, BSI tended to decline after the flare, suggesting changes in the BSI should be carefully observed to evaluate treatment with Ra-223 (Figure 4A and C).

The comparison between patients with a baseline BSI of $\geq 2.0$ and those with a baseline BSI of $<2$ using the KaplanMeier method showed that the OS of the latter group was significantly extended ( $p<0.05$; Figure 5). Moreover, our analysis also showed that the decrease in BSI was an independent factor of OS extension ( $p<0.05$; Table III). 


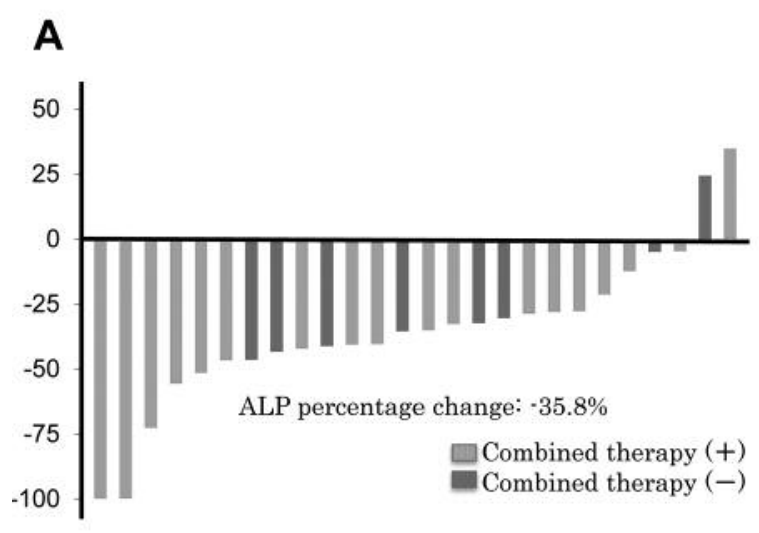

\section{B}

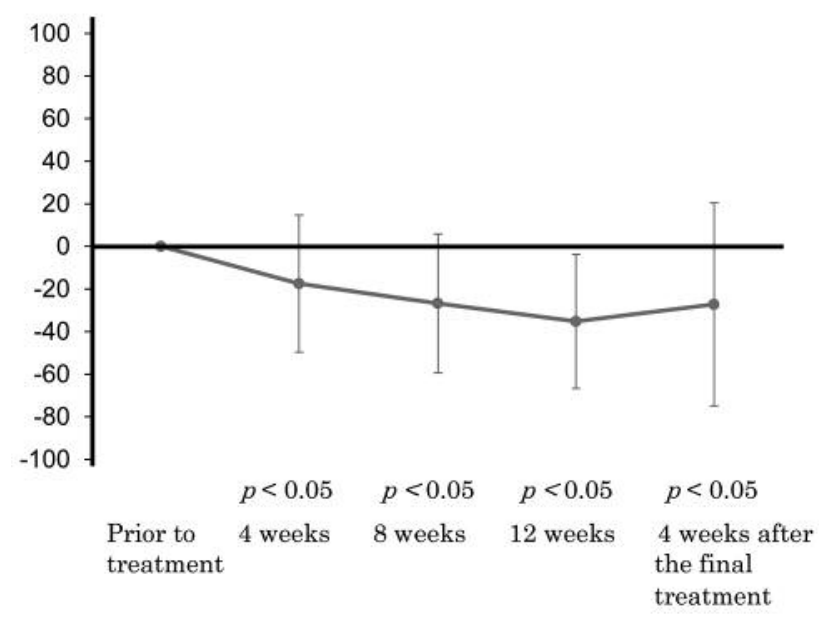

Figure 2. ALP changes from baseline with Ra-223 therapy. A: Percentage changes in ALP levels 12 weeks after treatment. Combined therapy was defined as concomitant treatment with "Ethinyl estradiol", "Enzalutamide" or "Abiraterone acetate+prednisolone". ALP: Alkaline phosphatase; PSA: prostate specific antigen. B: Percentage changes from baseline ALP (mean levels of ALP $\pm S D)$. ALP: Alkaline phosphatase; SD: standard deviation; PSA: prostate specific antigen.

\section{Discussion}

Ra-223 is a radioactive isotope with a physical half-life of 11.4 days that emits mainly alpha particles. However, due to its short range in tissue $(100 \mu \mathrm{m})$, the influence of Ra-223 on surrounding normal tissues (i.e., bone marrow) is considered to be limited (15). Therefore, Ra-223 may be useful in the treatment of CRPC patients with bone metastasis.

BSI is extremely useful in diagnosing bone metastases of prostate cancer (16). Although the flare occurred only once in the present study, BSI values tended to decline after the flare. This finding suggests that changes in BSI should be

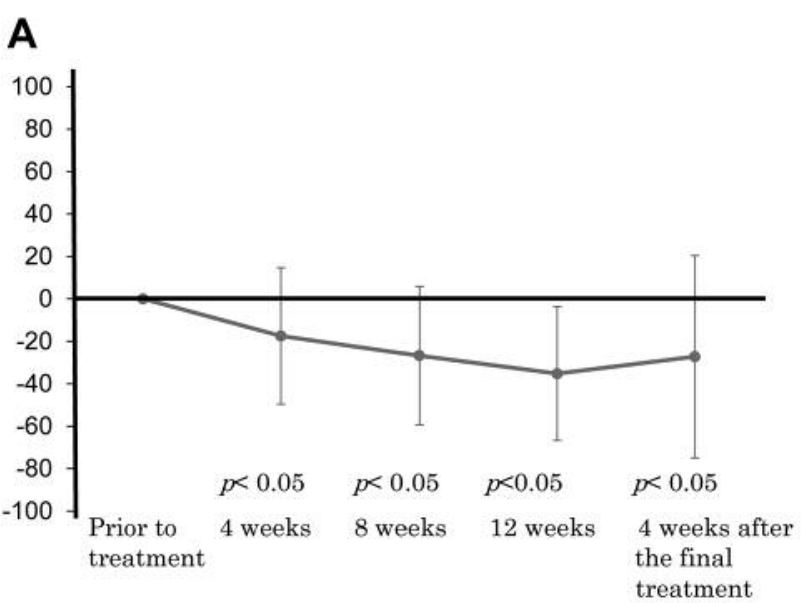

B

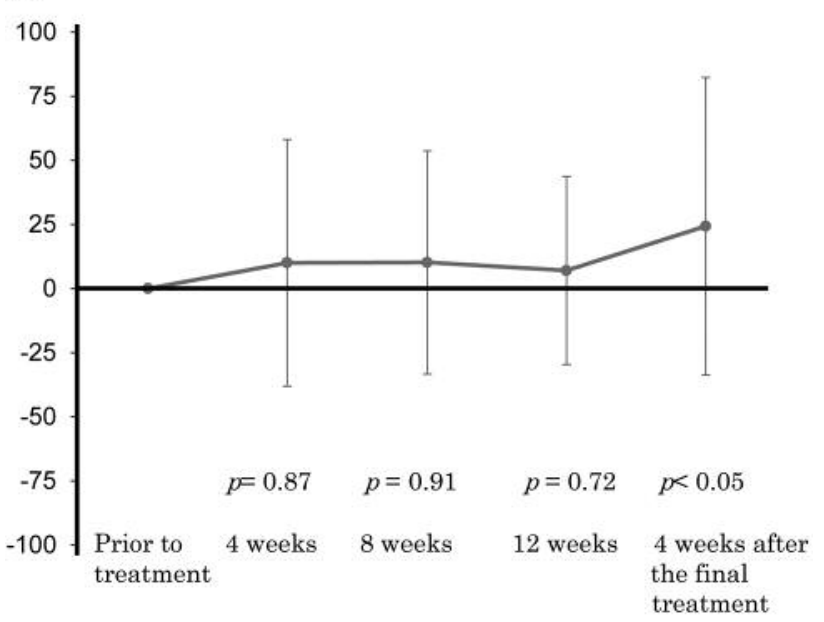

Figure 3. BAP and 1-CTP changes from baseline with Ra-223 therapy. $A$ : Percentage changes from baseline BAP (mean levels of $B A P \pm S D$ ). BAP: Bone type alkaline phosphatase; SD: standard deviation. B: Percentage changes in 1-CTP from baseline (mean levels of 1$C T P \pm S D)$. 1-CTP: Pyridinoline cross-linked carboxyterminal telopeptide of type 1 collagen; BAP: Bone type alkaline phosphatase; SD: standard deviation.

carefully observed to evaluate treatment with Ra-223 (Figure 4A, C). Furthermore, the observed decrease in BSI was an independent factor of OS extension (Table III). On the other hand, Alva et al. showed that baseline BSI values were significantly associated with OS in MCRPC patients treated with Ra-223 (17). In our study, the comparison between patients with a baseline BSI value of $\geq 2$ and those with a baseline BSI value of $<2$ using the Kaplan-Meier method showed that the OS in the latter group was significantly extended $(p<0.05)$ (Figure 5). Therefore, the baseline BSI value and the decrease in BSI following treatment are independent predictive factors of OS. 
A

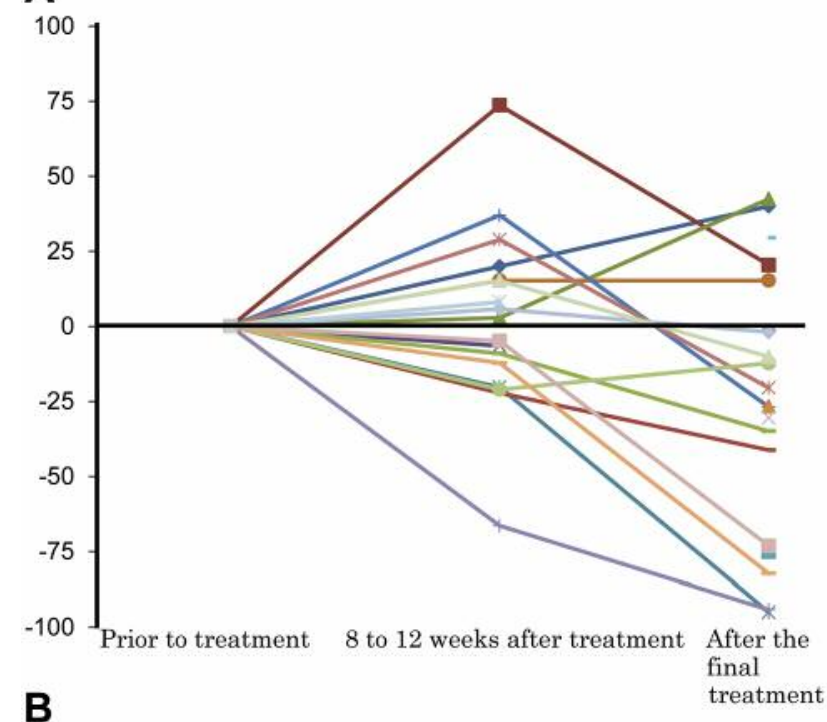

B

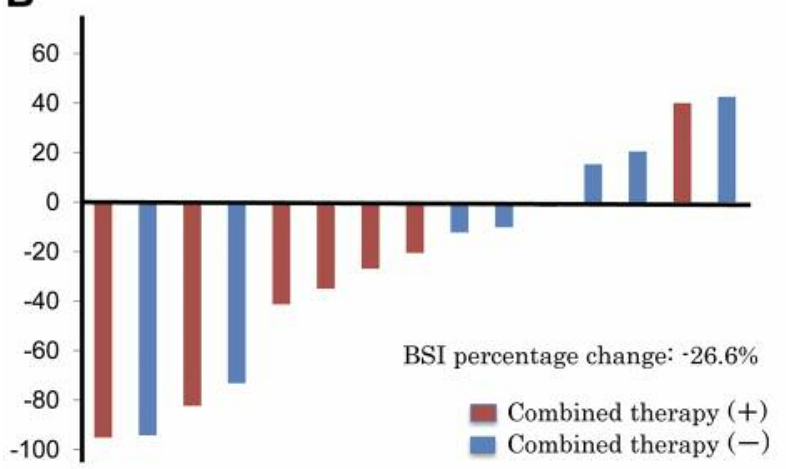

C

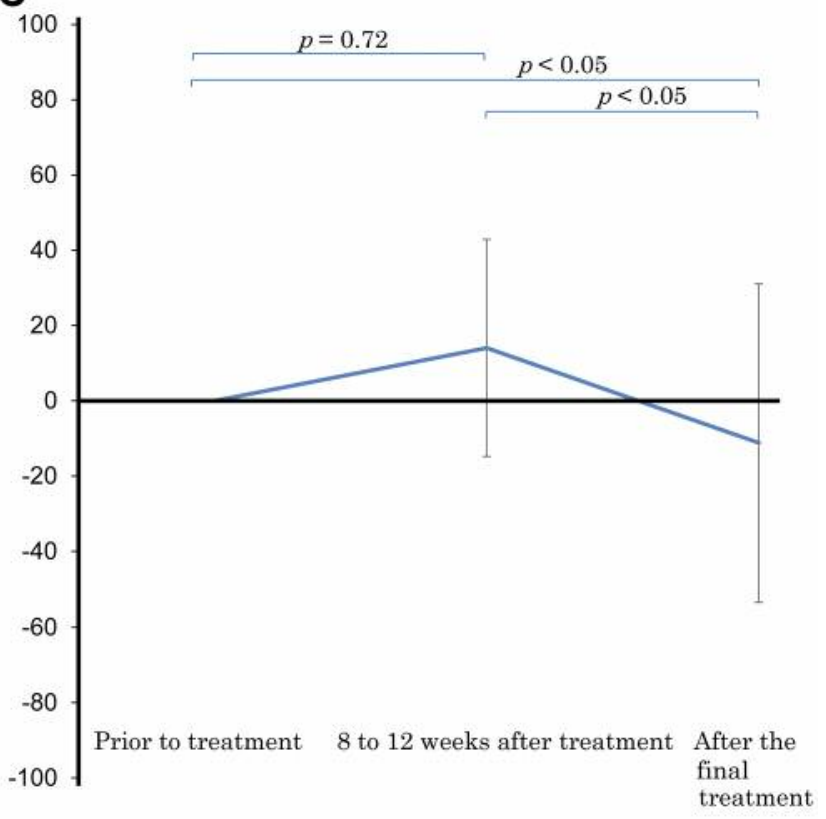

Figure 4. BSI changes from baseline with Ra-223 therapy. A: Percentage changes from baseline BSI. BSI: Bone scan index. B: Percentage changes in BSI levels after treatment. Combined therapy was defined as concomitant treatment with "Ethinyl estradiol", "Enzalutamide" or "Abiraterone acetate+prednisolone." BSI: Bone scan index. C: Percentage changes in BSI values (mean values of $B S I \pm S D)$. BSI: Bone scan index; SD, standard deviation.

Table III. Univariate and multivariate analyses of overall survival.

OS

OS

\begin{tabular}{|c|c|c|c|c|c|c|}
\hline \multirow[b]{2}{*}{ Data } & \multicolumn{3}{|c|}{ Univariate } & \multicolumn{3}{|c|}{ Multivariate } \\
\hline & HR & $95 \% \mathrm{CI}$ & $p$-Value & HR & $95 \% \mathrm{CI}$ & $p$-Value \\
\hline BSI value after the final treatment & 7.863 & $1.278-48.383$ & $<0.05$ & 17.798 & $1.066-297.179$ & $<0.05$ \\
\hline ALP 4 weeks after the final treatment & 0.687 & $0.138-30422$ & 0.647 & 1.015 & $0.076-13.517$ & 0.991 \\
\hline PSA 4 weeks after the final treatment & 0.667 & $0.146-3.043$ & 0.601 & 0.367 & $0.02-6.782$ & 0.501 \\
\hline BAP 4 weeks after the final treatment & 2.608 & $0.468-14.522$ & 0.274 & 0.631 & $0.024-16.501$ & 0.782 \\
\hline 1-CTP 4 weeks after the final treatment & 2.067 & $0.396-10.801$ & 0.389 & 2.372 & $0.362-15.527$ & 0.368 \\
\hline
\end{tabular}

In a domestic phase II clinical trial, the mean rate of change in PSA levels increased 12 weeks after treatment with Ra-223 compared to the baseline (7). Our study showed that the mean levels of PSA were significantly increased at 4 weeks after the final treatment (Figure 1B) compared with those reported at baseline (prior to treatment), and the rate of change in the PSA levels increased by $60.3 \%$ (Figure 1A). In addition, the mean levels of 1-CTP significantly increased 4 weeks after the final treatment compared with those observed at baseline $(p<0.05$; Figure $3 \mathrm{~B})$. Furthermore, in our multivariate analysis, PSA and 1-CTP were not shown to be independent factors for OS (Table III). These results 
suggest that the levels of PSA and 1-CTP are not suitable for monitoring the therapeutic effects of Ra-223.

Elevated levels of ALP have been associated with poor prognosis in patients with mCRPC (18-20). According to the same exploratory analysis on the ALSYMPCA trial, ALP levels decreased in approximately $80 \%$ of the patients in the Ra-223 group 12 weeks after treatment (21). Our study showed that the levels of ALP significantly decreased at 4, 8 , and 12 weeks, and continued to decrease until 4 weeks after the final treatment (all $p<0.05$; Figure 2B). The rate of change in the ALP levels 12 weeks after treatment decreased by $35.8 \%$ (Figure 2A). This decrease in ALP after treatment with Ra-223 is consistent with the results reported in the ALSYMPCA trial (21). In a case report, a patient with CRPC who received six intravenous doses of Ra-223 achieved bone pain relief and a significant decrease in the levels of BAP (22). In our study, the mean levels of BAP also significantly decreased from baseline BAP at each of the following measurement points: 4,8 , and 12 weeks after treatment, and 4 weeks after the final treatment (all $p<0.05$; Figure 3A). In the multivariate analysis, ALP and BAP were not shown to be independent factors for OS (Table III). Our results suggest that ALP and BAP levels may be useful for monitoring the therapeutic effects of Ra-223, but they are not predictive factors of OS.

In our study, the incidence of grade $\geq 3$ AEs (including anemia, nausea, and bone pain) was similar to that reported in the international phase III clinical trial ALSYMPCA (23). Furthermore, our results were consistent with those reported in a phase II clinical trial conducted in Japan (7). Overall, Ra-223 was well tolerated in Japanese CRPC patients with bone metastases.

In the ALSYMPCA trial involving 921 CRPC patients with bone metastases, the Ra-223 group (median OS, 14.9 months) showed an effectively extended median OS of 3.6 months (hazard ratio, 0.70 [95\% CI $=0.58-0.83$ ],$p<0.001$, stratified log-rank test) compared with the placebo group (median OS, 11.3 months) (23). Although the efficacy of Ra223 was only expected in bone metastases, the OS in CRPC patients was also extended, indicating the importance of local treatment in the bones.

In addition, results from the Phase III trial ERA 223 of Ra223 dichloride in combination with abiraterone acetate and prednisolone for patients with mCRPC were reported (24). Surprisingly, the incidence of bone fractures was increased in the $\mathrm{Ra}-223$ dichloride plus abiraterone acetate and prednisone arm of the study compared with that reported in the placebo plus abiraterone acetate and prednisolone arm, although in this study, the OS was also shorter in the Ra-223 dichloride plus abiraterone acetate and prednisolone arm of the study than in the placebo plus abiraterone acetate and prednisolone arm. In the ERA 223 study, subgroup analyses for the effect of bone health agents (BHA) (i.e., bisphosphonates or

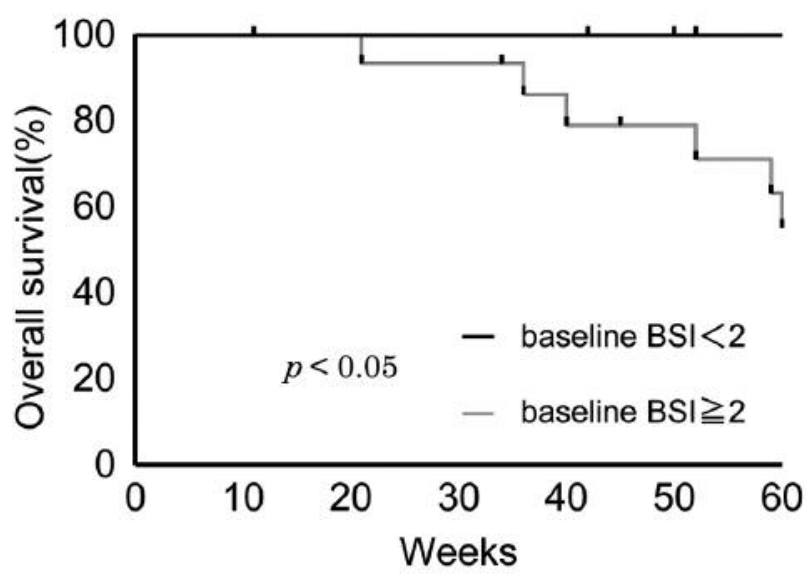

Figure 5. Kaplan-Meier curves showing changes in OS for each baseline BSI value. BSI: Bone scan index; OS: overall survival.

denosumab) were conducted for all fractures. These analyses showed that while treated with BHA, the incidence of symptomatic pathological bone fractures decreased in both the treatment and control arms. Based on this evidence, the use of BHA for fixing the bone environment is recommended in combination with Ra-223 treatment. Prednisolone is known to induce osteoporosis. Thus, worsening of the bone environment was expected in patients treated with Ra-223 dichloride plus abiraterone acetate and prednisolone. Presumably, the anticancer effects of abiraterone acetate and prednisolone with Ra-223 impaired bone environment and the conditions of the patients. Therefore, it is absolutely necessary to maintain bone health during Ra-223 treatment. Further investigations are necessary to determine the optimal combination of medicines for the prognosis and protection of the bone environment in CRPC patients receiving Ra-223 treatment.

In summary, Ra-223 was well tolerated in Japanese CRPC patients with bone metastases. AEs mainly included fatigue/malaise, gastrointestinal symptoms, diarrhea, and bone pain. Our study indicated that the levels of ALP and BAP (but not those of PSA and 1-CTP) are useful as evaluation markers for monitoring the therapeutic effects of Ra-223. Changes in BSI values, as well as ALP and BAP may also be useful for this purpose, despite the occurrence of flares. Moreover, it was shown that a baseline BSI values of $<2$ and the decrease in BSI after treatment are independent predictive factors of OS.

\section{Conflicts of Interest}

No potential conflicts of interest relevant to this article were reported. 


\section{Authors' Contributions}

Atsushi Mizokami: Conception and design, critical revision of the manuscript for important intellectual content, supervision. Shinro Matsuo: Acquisition of data, analysis and interpretation of data, drafting of the manuscript, statistical analysis. Tomoyuki Makino, Suguru Kadomoto, Hiroaki Iwamoto, Hiroshi Yaegashi, Masashi Iijima, Shohei Kawaguchi, Takahiro Nohara, Kazuyoshi Shigehara, Kouji Izumi, Yoshifumi Kadono: Administrative, technical or material support.

\section{References}

1 Scher HI, Halabi S, Tannock I, Morris M, Sternberg CN Carducci MA, Eisenberger MA, Higano C, Bubley GJ, Dreicer R, Petrylak D, Kantoff P, Basch E, Kelly WK, Figg WD, Small EJ, Beer TM, Wilding G, Martin A and Hussain M: Design and end points of clinical trials for patients with progressive prostate cancer and castrate levels of testosterone: Recommendations of the prostate cancer clinical trials working group. J Clin Oncol 26(7): 1148-1159, 2008. PMID: 18309951. DOI: 10.1200/ JCO.2007.12.4487

2 DePuy V, Anstrom KJ, Castel LD, Schulman KA, Weinfurt KP and Saad F: Effects of skeletal morbidities on longitudinal patient-reported outcomes and survival in patients with metastatic prostate cancer. Support Care Cancer 15(7): 869-876, 2007. PMID: 17262196. DOI: 10.1007/s00520-006-0203-x

3 Sathiakumar N, Delzell E, Morrisey MA, Falkson C, Yong M, Chia V, Blackburn J, Arora T, Brill I and Kilgore ML: Mortality following bone metastasis and skeletal-related events among women with breast cancer: A population-based analysis of u.S. Medicare beneficiaries, 1999-2006. Breast Cancer Res Treat 131(1): 231-238, 2012. PMID: 21842243. DOI: 10.1007/s10549-011-1721-x

4 Nilsson S, Strang P, Aksnes AK, Franzen L, Olivier P, Pecking A, Staffurth J, Vasanthan S, Andersson C and Bruland OS: A randomized, dose-response, multicenter phase ii study of radium223 chloride for the palliation of painful bone metastases in patients with castration-resistant prostate cancer. Eur J Cancer 48(5): 678686, 2012. PMID: 22341993. DOI: 10.1016/j.ejca.2011.12.023

5 Parker C and Sartor O: Radium-223 in prostate cancer. N Engl J Med 369(17): 1659-1660, 2013. PMID: 24152265. DOI: 10.1056/NEJMc1310231

6 Saad F, Carles J, Gillessen S, Heidenreich A, Heinrich D, Gratt J, Levy J, Miller K, Nilsson S, Petrenciuc O, Tucci M, Wirth M, Federhofer J and O'Sullivan JM: Radium-223 and concomitant therapies in patients with metastatic castration-resistant prostate cancer: An international, early access, open-label, single-arm phase 3b trial. Lancet Oncol 17(9): 1306-1316, 2016. PMID: 27473888. DOI: $10.1016 / \mathrm{S} 1470-2045(16) 30173-5$

7 Matsubara N, Nagamori S, Wakumoto Y, Uemura H, Kimura G, Yokomizo A, Kikukawa H, Mizokami A, Kosaka T, Masumori N, Kawasaki Y, Yonese J, Nasu Y, Fukasawa S, Sugiyama T, Kinuya S, Hosono M, Yamaguchi I, Tsutsui $\mathrm{H}$ and Uemura $\mathrm{H}$ : Phase ii study of radium-223 dichloride in japanese patients with symptomatic castration-resistant prostate cancer. Int J Clin Oncol 23(1): 173-180, 2018. PMID: 28770408. DOI: 10.1007/s10147017-1176-0

8 Soloway MS, Hardeman SW, Hickey D, Raymond J, Todd B, Soloway S and Moinuddin M: Stratification of patients with metastatic prostate cancer based on extent of disease on initial bone scan. Cancer 61(1): 195-202, 1988. PMID: 3334948.
9 Sadik M, Hamadeh I, Nordblom P, Suurkula M, Hoglund P, Ohlsson $\mathrm{M}$ and Edenbrandt L: Computer-assisted interpretation of planar whole-body bone scans. J Nucl Med 49(12): 1958-1965, 2008. PMID: 18997038. DOI: 10.2967/jnumed.108. 055061

10 Kikuchi A, Onoguchi M, Horikoshi H, Sjostrand K and Edenbrandt L: Automated segmentation of the skeleton in whole-body bone scans: Influence of difference in atlas. Nucl Med Commun 33(9): 947-953, 2012. PMID: 22760301. DOI: 10.1097/MNM.0b013e3283567407

11 Horikoshi H, Kikuchi A, Onoguchi M, Sjostrand $\mathrm{K}$ and Edenbrandt L: Computer-aided diagnosis system for bone scintigrams from japanese patients: Importance of training database. Ann Nucl Med 26(8): 622-626, 2012. PMID: 22729550. DOI: $10.1007 / \mathrm{s} 12149-012-0620-5$

12 Nakajima K, Edenbrandt L and Mizokami A: Bone scan index: A new biomarker of bone metastasis in patients with prostate cancer. Int J Urol, 2017. PMID: 28556293. DOI: 10.1111/ iju. 13386

13 Mitsui Y, Shiina H, Yamamoto Y, Haramoto M, Arichi N, Yasumoto $\mathrm{H}$, Kitagaki $\mathrm{H}$ and Igawa M: Prediction of survival benefit using an automated bone scan index in patients with castration-resistant prostate cancer. BJU Int 110(11 Pt B): E628-634, 2012. PMID: 22788759. DOI: 10.1111/j.1464-410X.2012. 11355.x

14 Anand A, Morris MJ, Larson SM, Minarik D, Josefsson A, Helgstrand JT, Oturai PS, Edenbrandt L, Roder MA and Bjartell A: Automated bone scan index as a quantitative imaging biomarker in metastatic castration-resistant prostate cancer patients being treated with enzalutamide. EJNMMI Res 6(1): 23, 2016. PMID: 26960325. DOI: 10.1186/s13550-0160173-z

15 Bruland OS, Nilsson S, Fisher DR and Larsen RH: High-linear energy transfer irradiation targeted to skeletal metastases by the alpha-emitter 223ra: Adjuvant or alternative to conventional modalities? Clin Cancer Res 12(20 Pt 2): 6250s-6257s, 2006. PMID: 17062709. DOI: 10.1158/1078-0432.CCR-06-0841

16 Mizokami A, Kadono Y, Kitagawa Y, Izumi K and Konaka H: Therapies for castration-resistant prostate cancer in a new era: The indication of vintage hormonal therapy, chemotherapy and the new medicines. Int J Urol 24(8): 566-572, 2017. PMID: 28577511. DOI: $10.1111 /$ iju. 13372

17 Alva A, Nordquist L, Daignault S, George S, Ramos J, Albany C, Isharwal S, McDonald M, Campbell G, Danchaivijitr P, Yentz S, Anand A and Yu EY: Clinical correlates of benefit from radium-223 therapy in metastatic castration resistant prostate cancer. Prostate 77(5): 479-488, 2017. PMID: 27990667. DOI: 10.1002/pros.23286

18 Chi KN, Kheoh T, Ryan CJ, Molina A, Bellmunt J, Vogelzang NJ, Rathkopf DE, Fizazi K, Kantoff PW, Li J, Azad AA, Eigl BJ, Heng DY, Joshua AM, de Bono JS and Scher HI: A prognostic index model for predicting overall survival in patients with metastatic castration-resistant prostate cancer treated with abiraterone acetate after docetaxel. Ann Oncol 27(3): 454-460, 2016. PMID: 26685010. DOI: 10.1093/annonc/mdv594

19 Cook RJ, Coleman R, Brown J, Lipton A, Major P, Hei YJ, Saad $\mathrm{F}$ and Smith MR: Markers of bone metabolism and survival in men with hormone-refractory metastatic prostate cancer. Clin Cancer Res 12(11 Pt 1): 3361-3367, 2006. PMID: 16740758. DOI:10.1158/1078-0432.CCR-06-0269

20 Lein M, Wirth M, Miller K, Eickenberg HU, Weissbach L, Schmidt K, Haus U, Stephan C, Meissner S, Loening SA and 
Jung K: Serial markers of bone turnover in men with metastatic prostate cancer treated with zoledronic acid for detection of bone metastases progression. Eur Urol 52(5): 1381-1387, 2007. PMID: 17321667. DOI: 10.1016/j.eururo.2007.02.033

21 Sartor O, Coleman RE, Nilsson S, Heinrich D, Helle SI, O'Sullivan JM, Vogelzang NJ, Bruland O, Kobina S, Wilhelm S, $\mathrm{Xu}$ L, Shan M, Kattan MW and Parker C: An exploratory analysis of alkaline phosphatase, lactate dehydrogenase, and prostate-specific antigen dynamics in the phase 3 alsympca trial with radium-223. Ann Oncol 28(5): 1090-1097, 2017. PMID: 28453701. DOI: $10.1093 / \mathrm{annonc} / \mathrm{mdx} 044$

22 Cabrera ME, Rey PM, Carrio I, Montes A and Lopez DA: Response to (223)ra-dichloride in castration-resistant prostate cancer with bone metastasis: A case report. Oncol Lett 12(2): 1323-1328, 2016. PMID: 27446432. DOI: 10.3892/ol.2016.4762

23 Parker C, Nilsson S, Heinrich D, Helle SI, O'Sullivan JM, Fossa SD, Chodacki A, Wiechno P, Logue J, Seke M, Widmark A, Johannessen DC, Hoskin P, Bottomley D, James ND, Solberg A, Syndikus I, Kliment J, Wedel S, Boehmer S, Dall'Oglio M, Franzen L, Coleman R, Vogelzang NJ, O'Bryan-Tear CG, Staudacher K, Garcia-Vargas J,
Shan M, Bruland OS and Sartor O: Alpha emitter radium-223 and survival in metastatic prostate cancer. N Engl J Med 369(3): 213223, 2013. PMID: 23863050. DOI: 10.1056/NEJMoa1213755

24 Smith M, Parker C, Saad F, Miller K, Tombal B, Ng QS, Boegemann M, Matveev V, Piulats JM, Zucca LE, Karyakin O, Kimura G, Matsubara N, Nahas WC, Nole F, Rosenbaum E, Heidenreich A, Kakehi Y, Zhang A, Krissel H, Teufel M, Shen J, Wagner $\mathrm{V}$ and Higano C: Addition of radium-223 to abiraterone acetate and prednisone or prednisolone in patients with castration-resistant prostate cancer and bone metastases (era 223): A randomised, double-blind, placebo-controlled, phase 3 trial. Lancet Oncol 20(3): 408-419, 2019. PMID: 30738780. DOI: $10.1016 / \mathrm{S} 1470-2045(18) 30860-\mathrm{X}$

Received March 11, 2019

Revised April 18, 2019

Accepted April 19, 2019 